\title{
The Main Morbid Gynaecological Conditions and Client Satisfaction towards the Provided Services in Ganayen District, Suez Governorate
}

\author{
Mona A. S. Attia, Hend A. Hassan, Mervat M. El Rafie \\ Public Health Department, Faculty of Medicine, Cairo University, Cairo 11562, Egypt \\ Corresponding author: Mona A. S. Attia, monaadelsoliman@cu.edu.eg
}

Received 14 December 2019;

Accepted 17 December 2019;

Published 20 December 2019

\begin{abstract}
Addressing women reproductive health $(\mathrm{RH})$ problems is an important topic on the global social agenda. Thus, the current study was conducted in 2017 to study the common morbid gynaecological conditions among Ganayen primary health care (PHC) centre and mobile clinic clients in Ganayen District, Suez Governorate, Egypt and assess client satisfaction towards the provided services. A sample of 300 females aged 15-49 years complaining of the common morbid conditions, which were identified by reviewing the two PHC facilities' records in the year 2016, and seeking medical advice from the studied PHC facilities were interviewed after receiving the service using a structured interview questionnaire and client satisfaction questionnaire, where the first 8 women who came to the centre or the clinic in the two days of work allocated for each facility were interviewed. Vaginal infection was the most common cause of seeking medical advice from the PHC centre while taking contraceptive method was the most common cause of seeking medical advice from the mobile clinic respectively. Both vaginal infection and uterine bleeding were common among hormonal contraception users. Follow-up appointments were given for only about half of women with recurrent vaginal infection. The clients were satisfied with the provided services with higher client satisfaction score for the PHC centre than the mobile clinic. Large-scale study should be conducted to explore the real situation prevailing in Egypt with objective assessment of the health facilities and health providers' performance. Also, training of health care providers on national management protocol of common morbid condition in addition to providing health education to raise the clients' awareness regarding these diseases are needed for proper management and prevention of recurrent and repeated attacks.
\end{abstract}

Keywords: Reproductive health, Gynaecological problems, Client satisfaction

\section{Introduction}

PHC represents an integral part of the country's health system. It is the first level of contact of individuals, family and community with the national health system conveying a comprehensive health care throughout the lifespan as close as possible to the everyday environment of people ${ }^{[1]}$.

Mobile clinics also represent an integral part of the healthcare system; it delivers care to populations that are hard to reach by the traditional system, improving access and supporting prevention and chronic disease management ${ }^{[2]}$.

There are more than 560 mobile clinics distributed in about 260 districts ${ }^{[3]}$. Suez is served by about 12 mobile units, distributed in the remote districts in the governorate.

Reproductive morbidity (RM) is defined as any morbidity which affects the reproductive tract, or which is a result of reproductive activities including sexual behaviour, pregnancy, abortion or child birth, it may also include morbidity of psychological nature. RM includes obstetric morbidity; including complications of pregnancy, labor, and puerperium; contraceptive morbidity as for example menorrhagia as a result of intra uterine device (IUD) use and thromboses as a complication of oral contraceptive use) and gynaecological morbidity as for example premenstrual syndrome, reproductive tract infection, , abnormal uterine bleeding ${ }^{[4]}$.

The most frequenting causes of gynaecological morbidity are vaginal infections which represent a major public health problem resulting in a diversity of problems that affect women at different ages followed by menstrual disorders ${ }^{[5]}$.

Addressing women RH problems is an important topic on the global social agenda for the coming century ${ }^{[6]}$.

$\mathrm{RH}$; as drawn by The International Conference on Population and Development; stays at the centre of development with a clear relation between RH, sustainable development and human rights. $\mathrm{RM}$ occurs frequently and it is seriously affects women's lives ${ }^{[7]}$.

Since the International Conference on Population and Development (ICPD), Stakeholders has been attentive to the RH situation in Egypt re-orienting policies and programs to provide adequate services, however, it is challenging. Bridging the gap in access to and utilization of RH care between urban areas from one side and rural or remote areas from the other side is a major 
challenge for North Africa including Egypt and Middle East region $^{[8]}$.

$\mathrm{RM}$ information is insufficient in developing countries, it is mainly based on data obtained from clinics and hospitals ${ }^{[9]}$; however, big proportion of clients does not visit such health facilities. Furthermore, the ability of women to accurately call to mind and state the manifestations that are correlated to complications' diagnoses is limited, mostly due to lack of specificity resulting in difficulties in estimating prevalence ${ }^{[10]}$ and reflecting the wrong value regarding the disease burden ${ }^{[11]}$.

The public primary level of care facilities is the only available opportunity for most low-income populations who constitute the mainstream of Egypt's population, thus improving the quality of services in primary health care facilities will improve the health status of a large proportion of the population.

Despite the availability of national quality assessment tool supplied by the Ministry of Health and Population, no data is available about the evaluation results of the mobile clinics and their role in providing services to females living in remote and low socioeconomic areas. In addition, although reproductive morbidity constitutes a serious public health problem in Egypt, it has remained an ignored area for public health research in remote areas. The aim of the current study is to identify the common morbid gynaecological conditions among women seeking medical advice from the PHC centers and mobile clinics and assess the client satisfaction regarding the provided services in Ganayen District, Suez Governorate.

\section{Materials and Methods}

\section{Study Design and Study Setting}

Health system operations research, exploratory study, the study employed a cross sectional design that was conducted in the primary health care center (El-Gabalayat family medicine unit) and in the mobile clinic (no: 276) in Ganayen district in Suez governorate.

\section{Sample Size and Sampling Technique}

Non-pregnant females in the reproductive age (15-49) who seek medical advice from the selected PHC center or mobile clinic for gynaecological problems in the two days per week allocated for data collection in each facility along the three months of data collection and agree to participate in the study were illegible to participate in the study.

A convenient sample of 300 females was identified at the beginning of the study; the sample size was calculated according to the statistical website "Raosoft" based on the following parameters: $5 \%$ margin of error, 95\% level of confidence, and about $50 \%$ prevalence of common morbid conditions among the facilities' clients as estimated from the facilities' records in the year before the study. (200) females were recruited from the PHC center and (100) females from the mobile clinic; as the mobile clinic is working only 2 days per week and the number of users is less than the PHC center, where the first 8 women who came to the center or clinic were interviewed. All the studied women were interviewed after taking the required services. (Exit interview)

\section{Data Collection Tools}

The facilities' records were reviewed to identify the most common causes of seeking medical advice in a one complete year (2016) before the study year (2017). Also, a structured questionnaire, by the researcher, including personal information, socio-demographic data, and cause of seeking medical advice from PHC center and mobile clinic and the currently used family planning method. It also includes a section for recording the preliminary diagnosis and the final diagnosis after investigations. Vaginal infection was classified clinically depending on the nature of the discharge while dysfunctional uterine bleeding (DUB) was classified according to the ultra-sonography (US) results. Also, a structured client satisfaction questionnaire which consists of 32 questions on service accessibility (7 points), applicability ( 8 points) and acceptability (5 points) and healthcare-providers efficiency (12 points) was used to assess the client satisfaction towards the provided services. Five possible responses were present for each question; the responses of "strongly agree", "agree" and "neutral", "disagree" and "strongly disagree" were scored 5, 4, 3, 2 and 1 respectively, with total maximum achieved score of 160 , the higher the client satisfaction score the more is their satisfaction with the provided service.

Client satisfaction score percent was calculated and interpreted as follows:

The achieved score from all answered questions X 100

The total score of whole questionnaire questions

More than or equal $85 \%$ is considered as excellent, from $75 \%$ to $85 \%$ is considered as very good, from $65 \%$ to $75 \%$ is interpreted as good, from $50 \%$ to $65 \%$ is considered as fair and less than is interpreted as $50 \%=$ bad

Then a mean of percent score of client satisfaction towards the facility either the PHC center or mobile clinic was estimated.

\section{Data Analysis}

Data were coded and entered on the computer using "Microsoft Office Excel Software "version 2007 then data was transferred and analyzed using Statistical Package for Social Science version 24 " SPSS v.24".

The data were summarized using frequency and percentage for qualitative variables. The mean and mean percent were used for calculation of client satisfaction mean score and mean score percent. Significance was tested using Mann-Whitney Test

\section{Ethical Consideration}

The study protocol was discussed by the staff members of the Public Health Department, Faculty of Medicine, Cairo University, and approved by its council held on 3/1/2017. Selected members of this department constituted the internal review board to guarantee the ethical conformity of the study and an approval from Research Ethics Committee was obtained.

Verbal consents were obtained from all participants before recruitment in the study, after explaining the objectives of the work. Confidentiality was guaranteed on handling the data base and questionnaire forms that were anonymous in accordance with the revised Helsinki decelerations of biomedical ethics ${ }^{[12]}$.

\section{Results}

Analysis of the socio-demographic characteristics of the study participants revealed that the mean age of studied women was $30.80 \pm 6.402$ years, most of them $(92.3 \%)$ achieved secondary level of education and 3\% university level. Housewives constituted $(95.7 \%)$ of all studied women. All the studied women are married and did not reach the menopause.

Table (1) shows the distribution of studied women by the cause of seeking medical advice from PHC center and mobile clinic; at PHC center, (58\%) came for vaginal infection treatment, 
(22\%) for Contraception taking, (11\%) for DUB treatment and (9\%) contraception change. While at mobile clinics; (58\%) came for contraception taking, (22\%) for Contraception change, (12\%) for DUB treatment and (8\%) for vaginal infections treatment.

Table 1: Percent distribution of studied women, by cause of seeking medical advice either from PHC center or mobile clinic

\begin{tabular}{|l|c|c|c|c|}
\hline \multirow{2}{*}{ Cause of seeking medical advice } & \multicolumn{2}{|c|}{ PHC Center (N=200) } & \multicolumn{2}{c|}{ Mobile Clinic (N=100) } \\
\cline { 2 - 5 } & Frequency & Percent(\%) & Frequency & \multicolumn{2}{c|}{ Percent(\%) } \\
\hline Vaginal infections & 116 & 58 & 58 & 58 \\
\hline Contraception taking & 44 & 22 & 22 & 22 \\
\hline Contraception change & 18 & 9 & 12 & 12 \\
\hline DUB & 22 & 11 & & \\
\hline
\end{tabular}

Table (2) shows that studied women with different types of vaginal infection are classified as follows: (56.5\%) of them had Candida and (27.4 $\%)$ had mixed infection and $(16.1 \%)$ had bacterial vaginosis. Only $43.7 \%$ of those having recurrent vaginal infections had taken follow up appointments.

Table 2: The percent distribution of different types of vaginal infection among the studied women complaining of vaginal infection

\begin{tabular}{|l|l|l|}
\hline Different types of vaginal infection & Frequency $\mathbf{( N = 1 2 4 )}$ & Percent (\%) \\
\hline Candida & 70 & 56.5 \\
\hline Bacterial vaginosis & 20 & 16.1 \\
\hline Mixed infection & 34 & 27.4 \\
\hline
\end{tabular}

Table (3) show that for only $(61.76 \%)$ of DUB cases US was done. The results of US were (47.6\%) have normal endometrium, (28.6\%) thick endometrium $(9.5 \%)$ has fibroid, $(9.5 \%)$ have ovarian mass/cyst and $(4.8 \%)$ have atrophic endometrium.

About one third (29.4\%) of the study participants having DUB are referred to specialist and (70.6\%) were not referred.

Table 3: Percent distribution of different causes of dysfunctional uterine bleeding according to the investigation (2D ultrasound) done among the participants of the study

\begin{tabular}{|l|l|l|}
\hline Investigation & Frequency N=(21) & Percent (\%) \\
\hline Thick Endometrium & 6 & 28.6 \\
\hline Atrophic endometrium & 1 & 4.8 \\
\hline Normal endometrium & 10 & 47.6 \\
\hline Fibroid & 2 & 9.5 \\
\hline Ovarian mass/cyst & 2 & 9.5 \\
\hline
\end{tabular}

Table (4) shows that $(65,3 \%)$ of the studied women having vaginal infection were hormonal contraception users and (34,7\%) were IUD users.

Also, it shows that $(58.8 \%)$ of DUB cases are hormonal contraception users and (38.3\%) of cases are related to IUD users and only (2.9\%) condom users and mean age of the studied women complaining of DUB was $30.63 \pm 6.089$ years.

Table 4: Pattern of contraception use among patients of vaginal infections and dysfunctional uterine bleeding

\begin{tabular}{|l|c|c|c|c|}
\hline \multirow{2}{*}{ Contraceptive method used } & \multicolumn{2}{|c|}{ Vaginal infection } & \multicolumn{2}{c|}{ Dysfunctional uterine bleeding } \\
\cline { 2 - 5 } & Frequency N=(124) & Percent $\mathbf{( \% )}$ & Frequency (N=34) & Percent $(\boldsymbol{\%})$ \\
\hline IUD & 43 & 34.7 & 13 & 38.3 \\
\hline Hormonal & 81 & 65.3 & 20 & 58.8 \\
\hline Condom & 0 & 0 & 1 & 2.9 \\
\hline
\end{tabular}

Table (5): shows the percent distribution of shifting in contraceptive use (Combined pills- Injectable 3 months - IUD - Mini-pillsInjectables1month- condom) among the studied women seeking medical advice from PHC center were 33.3\%, 27.8\%, 16.7\%, 16.7\%, 5.5\%, 0\% respectively. While the percent distribution of shifting in contraceptive use (IUD - Injectables 3 months -Combined pills - Mini-pillsInjectables1month- condom) among the studied women seeking medical advice in mobile clinic was $27.4 \%, 27.4 \%, 22.6 \%, 13.6 \%, 4.5 \%, 4.5 \%$ respectively.

Table 5: Percent distribution of studied women shifting their contraceptive method by the type of contraceptive method changed

\begin{tabular}{|l|c|c|c|c|}
\hline \multirow{2}{*}{ Type of contraceptive method changed } & \multicolumn{2}{|c|}{ PHC Center } & \multicolumn{2}{c|}{ Mobile Clinic } \\
\cline { 2 - 5 } & Frequency N=(18) & Percent (\%) & Frequency N=(22) & Percent (\%) \\
\hline IUD & 3 & 16.7 & 6 & 27.4 \\
\hline Combined pills & 6 & 33.3 & 5 & 22.6 \\
\hline Mini-pills & 3 & 16.7 & 3 & 13.6 \\
\hline Injectable 3 months & 5 & 27.8 & 6 & 27.4 \\
\hline Injectable 1 month & 1 & 5.5 & 1 & 4.5 \\
\hline Condom and others & 0 & 0 & 1 & 4.5 \\
\hline Total & $\mathbf{1 8}$ & $\mathbf{1 0 0}$ & $\mathbf{2 2}$ & $\mathbf{1 0 0}$ \\
\hline
\end{tabular}

Table (6): shows that achieved percent score of client satisfaction regarding; the accessibility, healthcare providers, applicability and acceptability of provided services from PHC center which were $(80.3 \%, 65 \%, 88.8 \%$ and $73.2 \%)$ respectively and the total client satisfaction to all services provided in PHC center is considered very good as it was (75.6\%). While, the achieved score of client satisfaction to the accessibility, healthcare providers, applicability and acceptability of provided services from mobile clinic were $(80.4 \%, 80 \%, 48.7 \%$ and $74 \%)$ 
respectively. And the total client satisfaction to all services provided in mobile clinic was good (73.9\%). The difference in client satisfaction between PHC center and mobile clinic is statistically significant $(\mathrm{p}<0.05)$.

Table 6: The achieved client satisfaction score regarding the services provided at PHC Center and Mobile clinic

\begin{tabular}{|c|c|c|c|c|c|c|c|}
\hline & \multicolumn{3}{|c|}{ PHC Center } & \multicolumn{3}{|c|}{ MOBILE CLINIC } & \multirow[t]{2}{*}{ P* value } \\
\hline & Mean & $\begin{array}{c}\text { Maximum } \\
\text { achieved mean }\end{array}$ & $\begin{array}{c}\text { Mean percent } \\
(\%)\end{array}$ & Mean & $\begin{array}{c}\text { Maximum } \\
\text { achieved mean }\end{array}$ & $\begin{array}{c}\text { Mean } \\
\text { percent }\end{array}$ & \\
\hline Accessibility of service & 28.1 & 35 & 80.3 & 20.1 & 25 & 80.4 & \\
\hline Healthcare-providers efficiency & 39.0 & 60 & 65 & 28.0 & 35 & 80 & \\
\hline Applicability of service & 35.5 & 40 & 88.8 & 7.3 & 15 & 48.7 & \\
\hline Acceptability of service & 18.3 & 25 & 73.2 & 11.1 & 15 & 74 & \\
\hline Total client satisfaction score & 120.9 & 160 & 75.6 & 66.5 & 90 & 73.9 & 0.001 \\
\hline
\end{tabular}

(*) Test of significance: Mann-Whitney Test.

\section{Discussion}

This study addresses a vital issue related to $\mathrm{RH}$ which is gynaecological morbidity, under the sustainable development goals, it is of utmost importance to think about the progress towards equitable coverage with reproductive health services. Therefore, the current study was designed in an attempt to provide a snap shot on the magnitude of the common morbid gynaecological condition in low-socioeconomic females seeking medical advice from either PHC center or mobile clinic in Ganayen district in Suez governorate and assess their satisfaction aiming to improve the quality of provided services in these primary health care facilities.

About $70 \%$ of the women seeking medical advice from PHC center were complaining from vaginal infection or DUB, while in the mobile clinic more than three quarters of participants seeking family planning services either contraceptive taking or change. This reflects the fact that most of women considered mobile clinics as family planning units; so the mean use is family planning settings.

Vaginal infection is considered one of the most frequent gynaecologic diagnoses encountered by physicians who provide primary care to women as reported in Gooch et al. study in $2011^{[13]}$. According to American Academy of Nurse practitioners (AANP) 2010, infectious vaginitis is experienced periodically by women during their reproductive lives because of its relationship with the menstrual cycles, aging, changes after pregnancy, birth control methods or medicines ${ }^{[14]}$.

In the current study, it was found that vaginal infection is the most common morbid complaint among the study participants (41.3\%) followed by DUB (11.3\%), a finding which is consistent with a study done in urban slums of Bhavnagar city in 2012, where reproductive tract infections (RTI) and menstrual problems were the most commonly diagnosed morbid conditions however, at different prevalence rate $(26.4 \%$ and $26 \%)$ respectively ${ }^{[15]}$, while the overall prevalence of vaginal infections in a study conducted in Ethiopia in 2015 was much less at $15.4 \%{ }^{[16]}$.

The global anaemia prevalence in 2011 which assumed that (29\%) of non-pregnant have anaemia ${ }^{[17]}$, making anaemia the commonest cause for maternal morbidity, however, this necessitates haemoglobin testing to confirm the diagnosis of anaemia; however, it is not frequently required laboratory test for non-pregnant women ${ }^{[18]}$.

Regarding the relation between the usage of contraception and vaginal infections, the current study revealed that two thirds of those diagnosed as having vaginal infection were hormonal contraception users $(65 \%)$ while about $(35 \%)$ were IUD users.

This finding can be explained by the contraceptives' content of Oestrogen and progesterone hormones that increase the vaginal glycogen exposing it to lactobacilli activities. It is widely believed that lactobacilli have a crucial role in glycogen conversion to lactic acid reducing the $\mathrm{pH}$ of the vagina. $\mathrm{PH}$ reduction leads to decrease in bacterial biota activities, thus favours the growth of yeasts including Candida species ${ }^{[19]}$.

These findings are consistent with those revealed from a case control study conducted in Bangladesh in 2007 among women of hormonal contraceptive users and non-contraceptive users, where Candida species (the most common species) was isolated from (56.7\%) of hormonal contraceptive users compared to $(31.1 \%)$ from non-contraceptive users ${ }^{[20]}$.

While this is inconsistent with a study addressing the prevalence of vaginal infection and studying the associated risk health behaviours among married women in 2015 in Ismailia city, the study found that more than half of patients with vaginal infection were IUD users ${ }^{[21]}$.

More than three quarters of those having vaginal infections were recurrent cases of vaginal infections $(77.4 \%)$ and only $(43.7 \%)$ of them had follow up appointment.

According to the Center for Disease Control (CDC), sexually transmitted diseases (STDs) treatment 2015, follow-up typically is not required, this is consistent with this study finding where no follow up appointment was given to those had no recurrent vaginal infection. However, women in whom symptoms persist or recur after initial treatment should be instructed to return back for follow-up visits which is inconsistent with the current study where only $(43.7 \%)$ of those had recurrent vaginal infection had taken follow up appointment.

US is an easy, safe, rapid and tolerable procedure it has an excellent diagnostic accuracy in the diagnosis of uterine pathology responsible for abnormal uterine bleeding, the study showed the distribution of different causes of DUB according to the ultrasound results as follows; normal endometrium, thick endometrium, fibroid, ovarian mass/cyst and atrophic endometrium at $47 \%, 28 \%$, $10 \%, 10 \%$ and $5 \%$ respectively and about one third of them were referred to specialist (29\%).

The high percentage of non-referral among DUB cases can be explained by the ultrasound results; where more than three quarters of the cases had normal endometrium and thick endometrium $(76 \%)$ that can be managed by general practitioner in the PHC center.

These findings were close to a study done among women in Upper Egypt, it documented that trans vaginal ultrasound results were as follows; no abnormality detected (NAD) in (44\%), fibroid in $(22 \%)$ ( 8 sub mucus, 14 interstitial), Poly cystic ovary (PCO) in $(15 \%)$, ovarian cyst in $(11 \%)$, and finally adenomyosis in $(8 \%)$ of cases $^{[22]}$.

Concerning pattern of contraception use among cases of DUB, the study demonstrated that more than half of the cases were 
hormonal contraception users $(58.8 \%)$, around one third were IUD users $(38.3 \%)$ and only $(2.9 \%)$ their husbands were condom users.

These findings are parallel to a study conducted by Moreau and his colleagues in 2007; it supposed that a lot of patients stop the use of hormonal contraceptives due to menstrual cycle disorders $^{[23]}$. Also, mini-pills and low-dose combined oral contraceptives are associated with a higher incidence of bleeding disturbances ${ }^{[23,24]}$ compared to non-hormonal contraceptives. According to Berenson study, the 3 months injectable "DepoProvera" is strongly linked with missed menstrual periods and bleeding for longer than 20 days ${ }^{[25]}$.

Regarding the quality of health services, a strong link between service quality and client satisfaction was proven by Smith and Swinehart in 2001 to the extent that "Quality is defined as perceived satisfaction in other consumer-oriented industries",[26] Patient satisfaction is an important component of the discrepancy between what is expected and what is perceived to be actually occurring. Thus, it is imperative to identify patient perceptions to assess the extent to which these ideals are met by their institutions ${ }^{[27]}$.

Attia and his colleagues study findings in $2005^{[28]}$, revealed that client satisfaction were related to geographical and financial accessibility and health providers' competency particularly the availability of female physicians. Also, Tucker and Adams study results in 2001 proved that the two dimensions that mostly shape patient satisfaction $^{[29]}$ were providers' performance and access variables. Provider performance is stated as the most significant factor in patient evaluations, linked with patient-caregiver interactions and interpersonal relations, whilst access variables are related to the patient's ability to get care and the impediments to that process.

The current study assessed client satisfaction regarding; the accessibility, healthcare providers efficiency, applicability and acceptability dimensions of provided services revealing a client satisfaction mean achieved score percent of $(80.3 \%, 65 \%, 88.8 \%$ and $73.2 \%$ ) respectively for the PHC center and $(80.4 \%, 80 \%$, $48.7 \%$ and $74 \%$ ) respectively for the mobile clinic.

The total client satisfaction score to all services provided in PHC center and mobile clinic were (very good $75.56 \%$ and good $73.6 \%)$ respectively. There was a statistically significant difference in the client satisfaction between PHC center and mobile clinic $(\mathrm{p}<0.05)$.

These high levels of client satisfaction can be explained by the low socio-economic status of the studied females. A finding that is parallel to a study that was conducted in Iran in 2016, the study addressed the relation between socio-economic status and client satisfaction with the public health care services, where the satisfied participants showed significantly lower levels of education than dissatisfied ones $(\mathrm{P}<0.001)$ and participants with low socio-economic level were significantly more satisfied than participants with high or middle socio-economic level $(\mathrm{P}<0.001)^{[30]}$.

\section{Conclusion and Recommendations}

Vaginal infections and abnormal uterine bleeding were the most common morbid gynaecological conditions identified where both vaginal infections and DUB were common with hormonal contraceptive users. More than half of the women seeking medical advice from PHC center were complaining from (vaginal infectionDUB), while in mobile clinics more than three quarters of participants seeking family planning services which reflects the fact that most of women considered mobile clinics as family planning units. The clients were satisfied from the services provided in both facilities with significant slightly higher client satisfaction score for the PHC center than the mobile clinic.

Large-scale study is needed to explore the real situation prevailing in Egypt. Also, preparation of health education messages for women is recommended to advise them on seeking medical care early and follow the recommended hygienic precautions to enjoy the benefits of healthy life together with planning and conducting training programs for health care providers on family planning, essential and common morbid conditions including the national management protocols.

\section{Limitations of the Study}

Some participants lack the ability to accurately recall and report signs or symptoms related to diagnoses. Also, some participants were embarrassed to talk about the bad aspects in the service provided either in the PHC and the mobile clinics.

\section{List of Abbreviations}

AANP: American Academy of Nurse practitioners

CDC: Center for Disease Control

DUB: Dysfunctional uterine bleeding

ICPD: International Conference on Population and Development

IUD: Intra uterine device

PHC: Primary health care

RH: Reproductive health

RM: Reproductive morbidity

RTI: Reproductive tract infections

STDs: Sexually transmitted diseases

US: Ultra-sonography

\section{Data availability}

Data are available and can be supplied by the author once requested.

\section{Conflict of interest}

All research authors have no conflict of interest of any type

\section{Funding Statement}

The study is self-funded

\section{Authors' contributions}

MA had a role in the conception and design of the study and drafting the article. HA had a role in data acquisition and analysis and drafting the article. ME had a role in interpretation of data, revising the article critically for important intellectual content. All authors read and approved the final manuscript.

\section{Acknowledgements}

Special thanks to the managers of the studied PHC facilities for facilitating the researchers' work.

\section{References}

[1] WHO, "Primary health care factsheet", 27 February 2019, https://www.who.int/news-room/fact-sheets/detail/ primary-health-care 
[2] C. F. Hill, B. W. Powers, S. H. Jain et al., "Mobile health clinics in the era of reform", Am J Manag Care. 2014 Mar; 20(3): 261-264.

[3] G. Al-Attar, "10 Years after introducing mobile clinics in Assuit, Egypt", Population Reference Bureau, 2009.

[4] WHO, "The consequences of maternal morbidity and mortality", a report of workshop, 1992.

[5] M. Dheresa, N. Assefa, Y. Berhaneet al., "Gynecological Morbidity among Women in Reproductive Age: A Systematic Review and Meta-Analysis", J Women's Health Care 6: 367. doi:10.4172/2167-0420.1000367, 2017.

[6] P. I. Kambo, B. S. Dhillon, P. Singh, B.N. Saxena, N.C. Saxena, "Self-Reported Gynaecological Problems from Twenty Three Districts of India", Indian Journal of Community Medicine, 28(2): 67-73, 2003.

[7] United Nations Population Fund, "Sexual and Reproductive Health " Overview", https://www.unfpa.org/sexual-reproductive-health, updated 16 November 2016.

[8] Population Reference Bureau, "Reproductive Health in the Middle East and North Africa- A Guide for Reporters", May 2008.

[9] C. Ronsmans, S. Scott, A. Adisasmita, P. Deviany, F. Nandiaty, "Estimation of population-based incidence of pregnancy-related illness and mortality (PRIAM) in two districts in West Java, Indonesia”, Br J Obstet Gynaecol. $116: 82-90,2009$.

[10] A. Montgomery, S. Goufodji, 1. Kanhonou et al., "Validity and reliability of postpartum morbidity questionnaires in Benin", Matern Child Health J., 16 : 1728-1731, 2012.

[11] M. M. Rahman, M. Kabin, M. Shahidullah, "Adolescent self-reported reproductive morbidity and health care seeking behaviour", Journal of Ayub Medical College Abbottabad, 16(2): 9-14, 2004.

[12] World Medical Association, "The Declaration of Helsinki. Bulletin of the World Health Organization"; 86: 650-651, 2008

[13] JW. Gooch, "Encyclopedia Dictionary of Polymers, 2nd edition, Springer Science and Business Media, LLC", 2011. Available at: http:// www. springer. com/la/book/9781441962461. (Accessed: 10 July 2017).

[14] American Academy of Nurse practitioners (AANP), "Health promotion, risk reduction and disease prevention", Journal of American Academy of nurse practitioners; 22: 57-9, 2010.

[15] V. Gosalia, P. Verma, V. Doshi et al., "Gynecological morbidities in women of reproductive age group in urban slums of Bhavnagar city", National Journal of Community Medicine, Volume 3, Issue 4:657-660, Oct Dec 2012

[16] W. Mulu, M. Yimer, Y. Zenebe, B. Abera, "Common causes of vaginal infections and antibiotic susceptibility of aerobic bacterial isolates in women of reproductive age attending at Felegehiwot Referral Hospital, Ethiopia: a cross sectional study", BMC Women's Health. 2015;15:42. Published 2015 May 13. doi:10.1186/s12905-015-0197-y
[17] Johns Hopkins Center for Communication Programs (CCP) and World Health Organization. Family Planning," A Global Handbook for Providers", 2011 Update Baltimore, MD: CCP; 2011.

[18] WHO, "The global prevalence of anaemia in 2011", World Health Organization 2015, ISBN 978924156496 0 (NLM classification: WH 155

[19] G. Donders, G. Bellen, D. Janssens et al., "Influence of contraceptive choice on vaginal bacterial and fungal microflora”, Eur J Clin Microbiol Infect Dis. 36(1):4348, 2017. doi: 10.1007/s10096-016-2768-8.

[20] M. A. Yusuf, M. A. K. Chowdhury, A. N. I. Sattar, M. M. Rahman, "Evaluation of the Effect of Contraceptives on the Prevalence of Candida Species on Vaginal Candidiasis in Dhaka, Bangladesh.”, Bangladesh Journal of Medical Microbiology, 1(2), 2007.

[21] Hayat, N. Shalaby, N. El-Maraghy, Z. Baraia, "Prevalence of vaginal infection and associated risk health behaviors among married women in Ismailia City.” Int. J. Curr. Microbiol. App. Sci 4(5): 555-567, 2015.

[22] E. Mahmoud, G. Abd El-Aziz, G. El-Darwish et al., "Perimenopausal bleeding in Upper Egypt." Int. J. Adv. Res. Biol.Sci. 1(7): 184-187, 2014.

[23] C. Moreau, K. Cleland, J. Trussell, "Contraceptive discontinuation attrib $\neg$ uted to method dissatisfaction in the United States.", Contraception. 76(4): 267-272, 2007.

[24] M. F. Gallo, K. Nanda, D. A. Grimes, L. M. Lopez, K. F. Schulz, "20 microg versus >20 microg estrogen combined oral contraceptives for contraception.", Cochrane Database Syst Rev; (4):CD003989, 2008.

[25] A. B. Berenson, S. D. Odom, C. R. Breitkopf, M. Rahman, "Physiologic and psychologic symptoms associated with use of injectable contracep $\neg$ tion and 20 microg oral contraceptive pills", Am J Obstet Gynecol; 199(4): 351.e1-351.e12, 2008.

[26] Smith, K. Swinehart, "Integrated systems design for customer focused health care performance measurement: A strategic service unit approach.”, International Journal of Health Care Quality Assurance. 2001; 14 (1): 21-28, 2001.

[27] V. S. Fan, M. Burman, McDonell, D Stephan, S. D. Fihn, "Continuity of care and other determinants of patient satisfaction with primary care", J Gen. Intern. med.; (20): 226-233, 2005.

[28] R. A. Attia, M. Abedel Razik, L. M. El-Grorory, H. Abou-Zienah, "Integration of PHC vertical program; implications and challenges", Faculty of medicine Cairo University, 2005.

[29] Tucker, S. R. Adams, "Incorporating patients' assessments of satisfaction and quality: an integrative model of patients' evaluations of their care", Managing Service Quality, 11(4): 272-86, 2001.

[30] N. Maharlouei, A. Mojtaba, M. Akbari, K. Lankarani, "Socioeconomic status and satisfaction with public healthcare system in Iran", Pub med, 2016. Available at: https:// www. ncbi. nlm. nih. gov/ pmc/ articles/PMC5219562/. (Accessed 28 August 2017). 